\title{
Unravelling neurogenetic networks implicated in developmental language disorders
}

\author{
Sonja C. Vernes and Simon E. Fisher ${ }^{\mathbf{1}}$
}

Wellcome Trust Centre for Human Genetics, University of Oxford, Roosevelt Drive, Oxford OX3 7BN, U.K.

\begin{abstract}
Childhood syndromes disturbing language development are common and display high degrees of heritability. In most cases, the underlying genetic architecture is likely to be complex, involving multiple chromosomal loci and substantial heterogeneity, which makes it difficult to track down the crucial genomic risk factors. Investigation of rare Mendelian phenotypes offers a complementary route for unravelling key neurogenetic pathways. The value of this approach is illustrated by the discovery that heterozygous FOXP2 (where FOX is forkhead box) mutations cause an unusual monogenic disorder, characterized by problems with articulating speech along with deficits in expressive and receptive language. FOXP2 encodes a regulatory protein, belonging to the forkhead box family of transcription factors, known to play important roles in modulating gene expression in development and disease. Functional genetics using human neuronal models suggest that the different FOXP2 isoforms generated by alternative splicing have distinct properties and may act to regulate each other's activity. Such investigations have also analysed the missense and nonsense mutations found in cases of speech and language disorder, showing that they alter intracellular localization, DNA binding and transactivation capacity of the mutated proteins. Moreover, in the brains of mutant mice, aetiological mutations have been found to disrupt the synaptic plasticity of Foxp2-expressing circuitry. Finally, although mutations of FOXP2 itself are rare, the downstream networks which it regulates in the brain appear to be broadly implicated in typical forms of language impairment. Thus, through ongoing identification of regulated targets and interacting co-factors, this gene is providing the first molecular entry points into neural mechanisms that go awry in language-related disorders.
\end{abstract}

\section{Introduction}

An array of childhood disorders affect aspects of language development, including SLI (specific language impairment) (MIM 606711) [1], DD (developmental dyslexia) (MIM 127700) [2], and ASDs (autism spectrum disorders) (MIM 209850) [3]. Although all of these language-related syndromes are highly heritable, the majority of cases show complex patterns of inheritance indicative of multifactorial aetiology, involving multiple risk factors and considerable genetic heterogeneity [4-6]. However, there are exceptional forms of such disorders which show a clear monogenic basis, and these rare cases can provide crucial new insights into the pathways that go awry during abnormal language development [7]. The most well studied example is the KE family, a multigeneration pedigree of over 30 members, approximately half of whom display severe deficits in speech and language development, whereas the remainder are unaffected [8]. The predominant feature of the disorder is a difficulty in mastering the complicated co-ordinated movements of lips, tongue, jaw

Key words: chromatin immunoprecipitation, contactin-associated protein-like 2 (CNTNAP2), forkhead box P2 (FOXP2), language, neurodevelopmental disorder, speech, transcription factor.

Abbreviations used: ChIP, chromatin immunoprecipitation; CNTNAP2, contactin-associated protein-like 2; DVD, developmental verbal dyspraxia; EMSA, electrophoretic mobility-shif assay; ENU, N-ethyl-N-nitrosourea; FOX, forkhead box; NLS, nuclear localization signal; polyQ, polyglutamine; SLI, specific language impairment; SNP, single nucleotide polymorphism.

${ }^{1}$ To whom correspondence should be addressed (email simon.fisher@well.ox.ac.uk). and soft palate that facilitate fluent speech, a condition known as DVD (developmental verbal dyspraxia) (MIM 602081) [8]. In the affected KE family members, their DVD is accompanied by impaired linguistic processing in expressive and receptive domains, affecting both spoken and written modalities [9]. There is considerable evidence that the disorder involves disturbed CNS (central nervous system) development and function. Structural neuroimaging in the KE family revealed subtle but significant correlates of the disorder, including reduced grey matter density in the inferior frontal gyrus (a region of the cortex) and caudate nucleus (part of the striatum) [10]. Moreover, in functional MRI (magnetic resonance imaging) experiments, the affected individuals displayed abnormal activation patterns during language-processing tasks, even when such tasks were performed silently, without speech output [11]. In particular, they showed underactivation of the left inferior frontal gyrus (cortex) and putamen (striatum).

A positional cloning study [12] eventually led to identification of the causative mutation in the KE family: all affected individuals carry a heterozygous point mutation (G-to-A transition) within exon 14 of a gene called FOXP2 (where FOX is forkhead box), located in chromosomal band 7q31 [13]. The protein encoded by FOXP2 belongs to a major group of transcription factors that share a characteristic type of DNAbinding domain, known as a Forkhead box [14]. The mutation in the KE family yields an arginine-to-histidine substitution altering a key residue $\left(\mathrm{Arg}^{553}\right)$ in the DNA-binding domain of 
Figure 1| Schematic diagram of the human FOXP2 locus, which spans over $600 \mathrm{~kb}$ in band 7q31

Black shading indicates translated exons; 'atg' and 'tga' denote positions of initiation and termination codons. The main FOXP2 protein isoform, encoded by exons $2-17$, contains 715 amino acids, with polyQ tracts of $40\left(Q_{40}\right)$ and ten residues $\left(\mathrm{Q}_{10}\right)$, a zinc-finger motif (ZnF), a leucine zipper (LeuZ), a forkhead domain (FOX) and an acidic C-terminus. Exons 3b and $4 a$ are alternatively spliced coding exons yielding amino acid insertions, whereas alternatively spliced exons $2 \mathrm{a}$, 2b and $3 \mathrm{a}$ are predicted to be non-coding. Exons s1-s3 and 1 represent alternative $5^{\prime}$-UTRs (untranslated regions). Exon 1 overlaps with a CPG island. Additional information on splicing and isoforms can be found in [20-22]. Sites of coding variants reported in cases of DVD are indicated below the schematic diagram, including the R553H mutation initially identified in the KE family [13], and three additional changes uncovered in a subsequent screening study of 49 other probands [31]. Also shown is the site of the translocation breakpoint found in case CS, mapping between exons $3 \mathrm{~b}$ and 4 . Several additional translocation cases involving FOXP2 disruption have since been reported [32-34]. Adapted from [31] with permission.

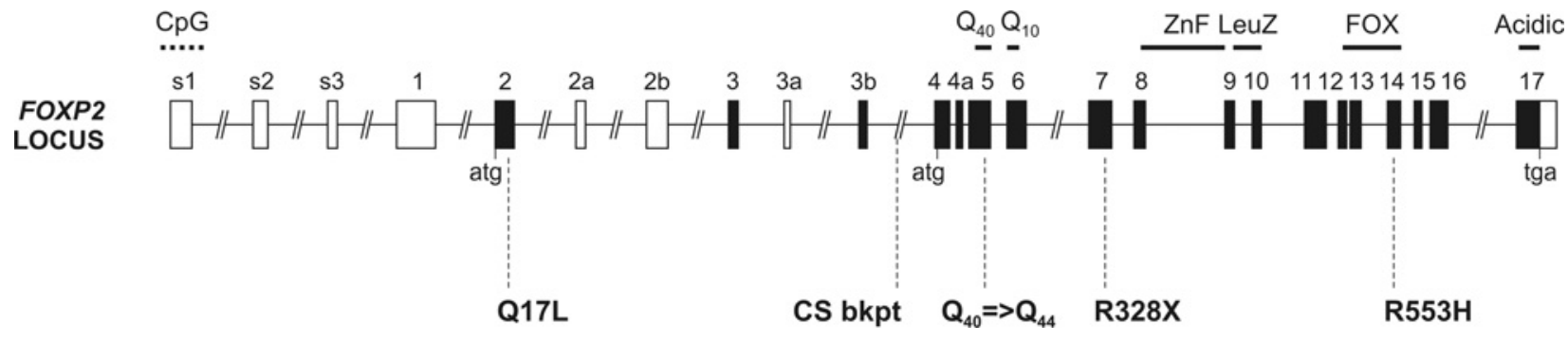

the FOXP2 protein (Figure 1). Indeed, this residue is invariant across all known FOX proteins, and the mutation was not present in any unaffected KE family individuals or in a large number of control chromosomes screened from the general population [13]. Additional evidence to connect this gene with speech and language impairment came from an unrelated case with a similar disorder. The child, known as CS, carried a de novo balanced translocation involving chromosomes 5 and 7 [15], with the site of chromosome 7 breakage directly disrupting the FOXP2 coding region (Figure 1) [13].

\section{Functional genetic analyses of FOXP2 isoforms}

FOX proteins are important regulators of eukaryotic gene expression, implicated in a range of developmental processes and disease states $[16,17]$. They have been classified into subgroups (named FOXA to FOXQ, in order of their discovery) based on comparing the amino acid sequences of their forkhead box DNA-binding domains. The FOXP subgroup is one of the most recent to have been defined and has a number of characteristics that set it apart from other FOX proteins [18]. The four different members of this subgroup show high levels of homology with each other [19]; for example, the three most closely related proteins (FOXP1, FOXP2 and FOXP4) display $\sim 92 \%$ amino acid similarity in their DNAbinding domains. However, these domains diverge from the typical forkhead box, in that they are truncated $(\sim 80$ amino acids rather than 100) and lie near to the C-terminal end of the protein. Other distinctive features of FOXP proteins include $\mathrm{N}$-terminal stretches that are rich in glutamine residues, as well as a conserved zinc-finger/leucine-zipper region that mediates dimerization (Figure 2) [18,19]. In fact, in contrast with other FOX transcription factors, which usually act as monomers, FOXPs are thought to bind DNA and regulate gene expression in homo- and hetero-dimeric form $[18,19]$.

Whereas the major transcript of human FOXP2 encodes a 715 amino acid protein product, the existence of multiple promoters and alternative splicing is predicted to yield several different isoforms (Figure 2), which may vary with cell type $[13,20,21]$. Functional studies in transfected cell lines, including neuronal models, investigated whether such isoforms display distinctive properties, assessing their subcellular localization, DNA-binding affinity and regulation of target genes [22]. The main form of the protein (isoform I) was compared with a C-terminally truncated isoform that contains dimerization domains, but lacks the DNA-binding motif (known as FOXP2.10+ or FOXP2-S), as well as an $\mathrm{N}$-terminally truncated isoform, lacking a portion of the glutamine-rich region (isoform III) (see Figure 2).

Isoform I protein was localized predominantly in the nucleus of cells, consistent with the idea that FOXP2 primarily functions as a transcription factor [22]. Putative NLSs (nuclear localization signals) were identified at the $\mathrm{N}$ - and C-terminal ends of the DNA-binding domain, and experiments using mutated/deleted expression constructs confirmed that these NLSs are indeed important for nuclear targeting of the protein $[22,23]$. Isoform III showed no deviation from the localization displayed by isoform I. In contrast, FOXP2.10+ protein, which lacks the DNAbinding domain, displayed markedly increased cytoplasmic localization, probably due to absence of the associated NLSs [22]. In addition, multiple independent reports have observed that cells transfected with FOXP2.10+ expression constructs typically develop intracellular aggregates of this protein isoform $[20,22,23]$. One such study demonstrated that the clusters of FOXP2.10+ protein co-localize with ubiquitin 
Figure 2 Representation of the full-length FOXP2 isoform I protein, containing the glutamine-rich region (Q-rich), zinc-finger (ZnF), leucine zipper (LeuZ) and forkhead-box (FOX) domains, and the C terminal acidic tail region (Acidic)

The predicted protein products yielded by coding changes identified in cases of DVD (R553H, Q17L, R328X) are aligned beneath. At the bottom, two isoforms of FOXP2 resulting from alternative splicing are shown. One isoform, referred to as FOXP2.10+, is encoded by an alternative mRNA transcript which contains a polyadenylation site in the intron following exon 10, and thus excludes exons 11-17 (see Figure 1). As such, the predicted product lacks the C-terminal regions containing the FOX and acidic domains, which are replaced by a short stretch of novel amino acids encoded by the slightly elongated version of exon 10. Another isoform, known as isoform III, is predicted to be translated from an internal initiation site in exon 4 (see Figure 1) from certain alternatively spliced transcripts, yielding a protein that is truncated at the $\mathrm{N}$-terminus by 92 amino acids. Adapted with permission from [22], which describes functional genetic analyses of each of the mutant products and alternative isoforms shown here.

FOXP2 Isoform I

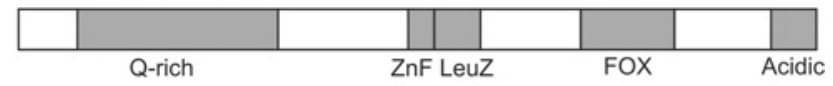

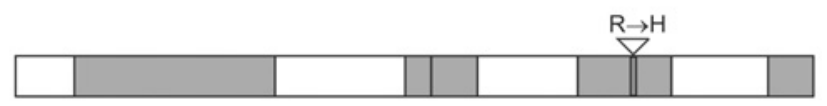

Coding
Changes $\left[\begin{array}{l}\text { FOXP2.R553H } \\ \text { FOXP2.Q17L } \\ \text { FOXP2.R328X }\end{array}\right.$

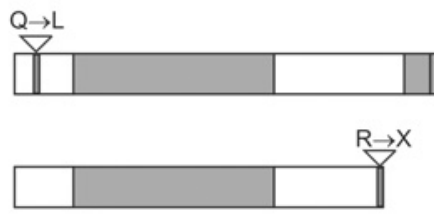

Splice
Variants $\left[\begin{array}{l}\text { FOXP2.10+ } \\ \text { FOXP2 Isoform III }\end{array}\right.$

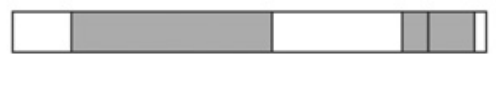

\begin{tabular}{|l|l|l|l|l|l|l|}
\hline & & & & & & \\
\hline
\end{tabular}

and $\gamma$-tubulin [22], classical markers of 'aggresomes', cytoplasmic (often perinuclear) bodies containing accumulations of misfolded protein [24]. Moreover, Western blotting showed that the FOXP2.10+ protein was itself ubiquitylated [22]. It is possible that these findings may relate to the presence of two polyQ (polyglutamine) tracts (one comprising 40 residues, followed by another comprising ten residues) in the glutamine-rich region of FOXP2, given the established link between aggresomes and polyQ diseases [24-26].

Although not yet fully understood, there is evidence to suggest that aggresomes may be able to sequester functional protein away from the site of action (e.g. the nucleus) and thus regulate the amount of active protein in a cell [24-26]. FOXP2.10+ retains the domains necessary for dimerization with other FOXP2 isoforms and FOXP subfamily members. Hence it is plausible that this truncated version of the protein could post-translationally regulate FOXP2 activity by forming dimers that localize to cytoplasmic aggresomes rather than the nucleus. Consistent with this hypothesis, despite lacking a DNA-binding domain, the FOXP2.10+ isoform was able to affect target gene expression in cell-based luciferase reporter assays, when expressed in the presence of endogenous wild-type FOXP2 [22].

\section{A range of FOXP2 mutations causing speech and language disorder}

Identification of the heterozygous $\mathrm{R} 553 \mathrm{H}$ missense mutation in the $\mathrm{KE}$ family and the translocation in case CS suggested that damage to one copy of FOXP2 is sufficient to derail speech and language development [13]. Indeed data from other $F O X$ genes show that cellular/developmental processes can be highly sensitive to the amounts of the functional transcription factor protein that are present $[16,17]$. Altered dosage of different $F O X$ genes has been implicated in major disease states, including developmental eye disorders (FOXC1) [27], ovarian failure (FOXL2) [28], immune deficiency (FOXP3) [29], and diabetes (FOXO1) [30]. A screening study assessed the wider contribution of FOXP2 mutations to developmental speech disorders, by analysing all of the known exons and flanking intronic sequence in 49 probands with a clinical diagnosis of DVD [31]. One of the probands was found to carry a heterozygous nonsense mutation (R328X) in exon 7 (Figure 1), which was predicted to yield dramatic truncation of the encoded FOXP2 protein, removing the zinc-finger/leucine-zipper region, the DNAbinding domain and other functional elements (Figure 2). The R328X mutation co-segregated with the disorder in 
the proband's family: it was present in a heterozygous state in his affected sister and his mother (who had a history of speech problems), but not found in his unaffected father, or in a large panel of control individuals from the general population [31]. Thus there was strong evidence that this was an aetiological mutation.

In the same screening study, two additional rare heterozygous variants were identified in independent probands: one case had a glutamine-to-leucine substitution mutation near the start of the protein (Q17L), whereas another had a slightly expanded polyQ tract, with 44 glutamine residues instead of 40 (Figure 1) [31]. The Q17L and Q40 $\rightarrow 44$ changes were not detected in screens of several hundred control chromosomes, indicating that they are not natural polymorphisms. However, in each case, the proband had a sibling diagnosed with DVD who did not carry the mutation [31]. Beyond point mutations, the literature now includes several reports of speech problems in children with heterozygous/hemizygous chromosomal rearrangements affecting the FOXP2 locus in $7 \mathrm{q} 31$, including translocations [32-34] and deletions $[32,35,36]$.

\section{Modelling the impact of aetiological FOXP2 mutations}

Genetic manipulation of human neuron-like cells has been used to assess the functional impact of the different FOXP2-coding variants found in people with DVD. Cells were transfected with constructs expressing mutated versions of FOXP2, corresponding to the R553H, R328X and Q17L variants (Figure 2) [22]. In these experiments, the Q17L change did not appear to disturb the functional properties of the FOXP2 protein, adding support to the view that it is unlikely to be an aetiological mutation [31]. In contrast, the R553H and R328X mutations each substantially affected multiple aspects of FOXP2 function, altering intracellular localization, DNA binding and transactivation capacity [22], but in distinct ways, as detailed below.

The $\mathrm{R} 553 \mathrm{H}$ substitution replaces a highly conserved arginine residue located within the recognition helix of FOXP2's DNA-binding domain [13]. Indeed, X-ray crystallography studies suggest that the arginine residue in question normally makes direct contact with the target DNA, and also mediates intraprotein interactions that help maintain the three-dimensional structure of the domain [37]. EMSAs (electrophoretic mobility-shift assays) demonstrated that substituting a histidine residue for this arginine residue of the FOXP2 protein abolishes binding to its core DNA recognition site [22]. Moreover, luciferase-based reporter assays showed that this $\mathrm{R} 553 \mathrm{H}$ substitution significantly interferes with the protein's ability to regulate target gene expression [22]. The $\mathrm{R} 553 \mathrm{H}$ variant was also found to display a partial reduction of nuclear localization $[22,23]$, albeit in a much more subtle manner than that observed for paralogous changes in other FOX genes [27].

Thus the results of cell-based assays support the idea that the $\mathrm{R} 553 \mathrm{H}$ mutation yields a loss of function, such that the disorder in the affected members of the KE family (all of whom are heterozygotes) would result from a half dosage of functional FOXP2. Nevertheless, a number of findings hint at the possibility of an additional dominant negative effect in these heterozygotes, given that the dysfunctional protein product still retains dimerization domains and the potential to interact with wild-type FOXP2 (or other FOXP proteins in sites of co-expression). For example, the degree of nuclear targeting of the $\mathrm{R} 553 \mathrm{H}$ protein has been noted to vary in relation to the levels of endogenous wild-type FOXP2 in the cell [22]. Conversely, when cells are co-transfected with recombinant $\mathrm{R} 553 \mathrm{H}$ and wild-type FOXP2, the presence of the mutant product appears to affect intracellular localization of the normal protein [23]. Finally, data from luciferase assays suggest that overexpression of the $\mathrm{R} 553 \mathrm{H}$ variant can disturb the transactivation capabilities of endogenous FOXP proteins [22]. It is worth noting, however, that the above experiments involved overexpression of recombinant proteins in cell culture; it remains to be determined whether dominant-negative effects have physiological relevance in vivo in the brains of $\mathrm{R} 553 \mathrm{H}$ heterozygotes.

The R328X nonsense mutation [31] yields a C-terminally truncated version of FOXP2 protein, which appears to be unstable; in cell-based studies, very low levels of this product were observed even during overexpression assays [22]. The truncated protein was primarily located in the cytoplasm rather than the nucleus, and it showed no ability to bind or regulate target DNA [22]. These findings are explained by the fact that it lacks both the dimerization motif and the DNA-binding domain (which also contains the major NLSs). Consistent with the loss of the dimerization region, there was no evidence of any dominant-negative effects on endogenous FOXP proteins. Thus the R328X mutation yields an essentially non-functional transcription factor. Further work is needed to establish whether the distinct properties of the $\mathrm{R} 553 \mathrm{H}$ and $\mathrm{R} 328 \mathrm{X}$ variants in cell-based assays are reflected by differences in phenotypic profile of people who carry these mutations. Clearly each causes a disorder that is primarily characterized by DVD and impaired linguistic function [13,31], but detailed comparisons of behavioural/cognitive measures have not yet been made.

Studies of animal models are giving important new insights into the in vivo impact of these aetiological mutations [38]. Foxp2, the mouse orthologue of FOXP2, is highly homologous with its human counterpart; the major protein isoforms encoded by the Mus musculus and Homo sapiens genes differ by only three amino acid substitutions and a minor change in polyQ tract length. FOXP2/Foxp2 is expressed in similar neural structures in the two species, with enrichment in corresponding neuronal subpopulations, including deep layers of the cortex, dorsal nuclei in the thalamus, medium spiny neurons of the striatum and Purkinje cells in the cerebellum [39]. An allelic series of mice carrying different Foxp2 point mutations was generated via gene-driven ENU ( $N$-ethyl- $N$-nitrosourea) screening [40]. Crucially the ENU mutations included Foxp2-R552H, which matches the human FOXP2-R553H mutation (the difference 
in numbering relates to the slightly shorter polyQ length of mouse Foxp2) and Foxp2-S321X, which truncates the protein at a position that is very close to the human FOXP2-R328X mutation. Analyses of brain tissue from S321X mutants indicated that this allele is effectively null for Foxp 2 protein, probably due to a combination of nonsense-mediated RNA decay and instability of truncated product [40].

Mice that were homozygous for mutant alleles showed severe developmental delays, general motor dysfunction, delayed maturation of the cerebellum and lethality at approx. 3-4 weeks after birth. In contrast, heterozygotes (matching the heterozygous status of humans with the disorder) were healthy and developed normally, with no obvious anomalies in brain structure or baseline motor behaviour. Detailed studies of the R552H ENU heterozygotes found that they had impaired motor skill learning on running wheels and accelerating rotarods [40]. This was accompanied by significant alterations in synaptic plasticity in relevant Foxp2-expressing neural circuits; in particular, there was an absence of long-term depression in the dorsolateral striatum [40]. Further understanding of the impact of Foxp2 disruptions on neural plasticity and the relationship with behavioural phenotypes will require use of conditional alleles in which the gene can be selectively ablated in specific neural structures and circuits [41], especially since it is also expressed in a number of non-neural tissues [13].

\section{Defining downstream pathways regulated by FOXP2}

Given that FOXP2 encodes a transcription factor, it provides an ideal entry point into neurogenetic pathways associated with speech and language [7,38]. Although mutations of FOXP2 itself are rare, causing a rather unusual type of speech and language disorder, it is plausible that the downstream networks which it regulates in the brain are more broadly implicated in common forms of impairment, such as SLI (defined as a failure to acquire normal expressive/receptive language despite adequate environmental influences and in the absence of other medical/genetic causes). Evidence to support the hypothesis came from a study by Vernes et al. [42] in which ChIP (chromatin immunoprecipitation) was coupled to shotgun cloning and sequencing, to identify genomic regions bound by FOXP2 protein in a human neuronal model. This process uncovered a FOXP2bound fragment located within the first intron of the human CNTNAP2 (contactin-associated protein-like 2) gene, encoding a member of the neurexin superfamily of transmembrane proteins. EMSAs confirmed strong specific binding of FOXP2 to consensus target sites within the fragment, while quantitative RT (reverse transcription)-PCR experiments indicated that increased FOXP2 expression led to significantly reduced mRNA levels for multiple isoforms of CNTNAP2 [42]. Consistent with this repressive effect, the two genes show complementary expression with respect to cortical layering in human foetal brain at mid-gestation [42].
In addition to being regulated by FOXP2 [42], the CNTNAP2 target is implicated in important processes in brain development, including cell adhesion, neuronal recognition and localization/maintenance of voltage-gated potassium channels [43], it shows enriched expression in frontal grey matter in developing cerebral cortex [44] and it is associated with disorders such as epilepsy and autism [44,45]. Therefore Vernes and colleagues reasoned that it was a particularly promising candidate to test for involvement in language-related phenotypes. They therefore genotyped SNPs (single nucleotide polymorphisms) spanning CNTNAP2 in a set of 184 small nuclear families affected with typical SLI [42], part of an ongoing U.K.-based collection established by the SLI consortium [46]. Significant association was observed between a cluster of SNPs located in the exon 13-15 region of CNTNAP2 and reduced language performance in the SLI families [42]. This was particularly evident for a key quantitative endophenotype, a reduced ability to repeat pronounceable nonsense words, which is thought to index impairments in phonological short-term memory, one of the core deficits in SLI [6]. Intriguingly, SNPs in the same region of CNTNAP2 have also been associated with language delays in male autistic probands [44]. Since cases of autism were rigorously excluded from the SLI collection in the study by Vernes et al. [42], it seems that CNTNAP2 risk variants may influence language-related endophenotypes that span traditional diagnostic boundaries. Overall, this work points to mechanistic links between a rare Mendelian speech and language disorder (caused by FOXP2 mutation) and common genetically complex forms of language impairment (influenced by polymorphisms in CNTNAP2, a direct neural target of FOXP2) [42].

So far, CNTNAP2 is the only FOXP2 target to have been investigated for involvement in language impairments. Nevertheless, FOXP2, like other forkhead transcription factors, regulates a large number of different genes, which might vary from one cell type to another depending on the profile of co-factors that are present. High-throughput searches for directly regulated target genes have been carried out by coupling FOXP2-ChIP with screening of microarrays (ChIP-on-chip) in human fetal brain samples and neuronal models $[47,48]$. By using material purified via ChIP to interrogate $\sim 5000$ human promoters, the studies identified several hundred putative target genes, with significant overlap between cellbased and in vivo findings. A representative subset of the potential targets was chosen for further validation on a geneby-gene basis using techniques such as EMSA and qRTPCR (quantitative reverse transcription-PCR). In each study, most of the validated targets were repressed by FOXP2, but a small number of cases appeared to show activation $[47,48]$. Gene ontology analyses identified several interesting biological themes in the downstream networks highlighted by these investigations, with target gene lists showing significant overrepresentations of categories such as axon guidance, cell adhesion, Wnt signalling and synaptic plasticity [47,48].

Full genome-wide screens for FOXP2 binding are underway, along with expression profiling of neuronal 
subpopulations in model systems, which should yield a fuller understanding of the functions of this gene. Moreover, analyses of co-factors that interact with FOXP2 might provide further clues to its involvement in speech and language functions [49]. Finally, it is worth noting that functional analyses of FOXP2 will not only shed light on the contributions of the gene to aetiology of neurodevelopmental disorders, but may also answer more fundamental questions regarding the origins of human speech and language [50].

\section{Funding}

We gratefully acknowledge financial support from the Royal Society, Wellcome Trust and Autism Speaks. S.E.F. is a Royal Society Research Fellow.

\section{References}

1 Fisher, S.E., Lai, C.S. and Monaco, A.P. (2003) Deciphering the genetic basis of speech and language disorders. Annu. Rev. Neurosci. 26, 57-80

2 Fisher, S.E. and DeFries, J.C. (2002) Developmental dyslexia: genetic dissection of a complex cognitive trait. Nat. Rev. Neurosci. 3, 767-780

3 Tager-Flusberg, H., Joseph, R. and Folstein, S. (2001) Current directions in research on autism. Ment. Retard. Dev. Disabil. Res. Rev. 7, 21-29

4 Abrahams, B.S. and Geschwind, D.H. (2008) Advances in autism genetics: on the threshold of a new neurobiology. Nat. Rev. Genet. 9, 341-355

5 Fisher, S.E. and Francks, C. (2006) Genes, cognition and dyslexia: learning to read the genome. Trends Cogn. Sci. 10, 250-257

6 Newbury, D.F., Bishop, D.V. and Monaco, A.P. (2005) Genetic influences on language impairment and phonological short-term memory. Trends Cogn. Sci. 9, 528-534

7 Fisher, S.E. (2007) Molecular windows into speech and language disorders. Folia Phoniatr. Logop. 59, 130-140

8 Hurst, J.A., Baraitser, M., Auger, E., Graham, F. and Norell, S. (1990) An extended family with a dominantly inherited speech disorder. Dev. Med. Child Neurol. 32, 352-355

9 Watkins, K.E., Dronkers, N.F. and Vargha-Khadem, F. (2002) Behavioural analysis of an inherited speech and language disorder: comparison with acquired aphasia. Brain 125, 452-464

10 Watkins, K.E., Vargha-Khadem, F., Ashburner, J., Passingham, R.E., Connelly, A., Friston, K.J., Frackowiak, R.S., Mishkin, M. and Gadian, D.G. (2002) MRI analysis of an inherited speech and language disorder: structural brain abnormalities. Brain 125, 465-478

11 Liegeois, F., Baldeweg, T., Connelly, A., Gadian, D.G., Mishkin, M. and Vargha-Khadem, F. (2003) Language fMRI abnormalities associated with FOXP2 gene mutation. Nat. Neurosci. 6, 1230-1237

12 Fisher, S.E., Vargha-Khadem, F., Watkins, K.E., Monaco, A.P. and Pembrey, M.E. (1998) Localisation of a gene implicated in a severe speech and language disorder. Nat. Genet. 18, 168-170

13 Lai, C.S., Fisher, S.E., Hurst, J.A., Vargha-Khadem, F. and Monaco, A.P. (2001) A forkhead-domain gene is mutated in a severe speech and language disorder. Nature 413, 519-523

14 Cirillo, L.A. and Barton, M.C. (2008) Many forkheads in the road to regulation: symposium on forkhead transcription factor networks in development, signalling and disease. EMBO Rep. 9, 721-724

15 Lai, C.S., Fisher, S.E., Hurst, J.A., Levy, E.R., Hodgson, S., Fox, M., Jeremiah, S., Povey, S., Jamison, D.C., Green, E.D. et al. (2000) The SPCH1 region on human 7q31: genomic characterization of the critical interval and localization of translocations associated with speech and language disorder. Am. J. Hum. Genet. 67, 357-368

16 Carlsson, P. and Mahlapuu, M. (2002) Forkhead transcription factors: key players in development and metabolism. Dev. Biol. 250, 1-23

17 Lehmann, 0.J., Sowden, J.C., Carlsson, P., Jordan, T. and Bhattacharya, S.S. (2003) Fox's in development and disease. Trends Genet. 19, 339-344

18 Wang, B., Lin, D., Li, C. and Tucker, P. (2003) Multiple domains define the expression and regulatory properties of Foxp1 forkhead transcriptional repressors. J. Biol. Chem. 278, 24259-24268

19 Li, S., Weidenfeld, J. and Morrisey, E.E. (2004) Transcriptional and DNA binding activity of the Foxp $1 / 2 / 4$ family is modulated by heterotypic and homotypic protein interactions. Mol. Cell. Biol. 24, 809-822
20 Bruce, H.A. and Margolis, R.L. (2002) FOXP2: novel exons, splice variants, and CAG repeat length stability. Hum. Genet. 111, 136-144

21 Schroeder, D.I. and Myers, R.M. (2008) Multiple transcription start sites for FOXP2 with varying cellular specificities. Gene 413, 42-48

22 Vernes, S.C., Nicod, J., Elahi, F.M., Coventry, J.A., Kenny, N., Coupe, A.M., Bird, L.E., Davies, K.E. and Fisher, S.E. (2006) Functional genetic analysis of mutations implicated in a human speech and language disorder. Hum. Mol. Genet. 15, 3154-3167

23 Mizutani, A., Matsuzaki, A., Momoi, M.Y., Fujita, E., Tanabe, Y. and Momoi, T. (2007) Intracellular distribution of a speech/language disorder associated FOXP2 mutant. Biochem. Biophys. Res. Commun. 353 869-874

24 Kopito, R.R. (2000) Aggresomes, inclusion bodies and protein aggregation. Trends Cell Biol. 10, 524-530

25 Tanaka, M., Kim, Y.M., Lee, G., Junn, E., Iwatsubo, T. and Mouradian, M.M. (2004) Aggresomes formed by $\alpha$-synuclein and synphilin-1 are cytoprotective. J. Biol. Chem. 279, 4625-4631

26 Webb, J.L., Ravikumar, B. and Rubinsztein, D.C. (2004) Microtubule disruption inhibits autophagosome-lysosome fusion: implications for studying the roles of aggresomes in polyglutamine diseases. Int. J. Biochem. Cell Biol. 36, 2541-2550

27 Saleem, R.A., Banerjee-Basu, S., Berry, F.B., Baxevanis, A.D. and Walter, M.A. (2003) Structural and functional analyses of disease-causing missense mutations in the forkhead domain of FOXC1. Hum. Mol. Genet. 12, 2993-3005

28 Crisponi, L., Deiana, M., Loi, A., Chiappe, F., Uda, M., Amati, P., Bisceglia, L., Zelante, L., Nagaraja, R., Porcu, S. et al. (2001) The putative forkhead transcription factor FOXL2 is mutated in blepharophimosis/ptosis/ epicanthus inversus syndrome. Nat. Genet. 27, 159-166

29 Lopes, J.E., Torgerson, T.R., Schubert, L.A., Anover, S.D., Ocheltree, E.L., Ochs, H.D. and Ziegler, S.F. (2006) Analysis of FOXP3 reveals multiple domains required for its function as a transcriptional repressor. J. Immunol. 177, 3133-3142

30 Nakae, J., Biggs, 3rd, W.H., Kitamura, T., Cavenee, W.K., Wright, C.V., Arden, K.C. and Accili, D. (2002) Regulation of insulin action and pancreatic $\beta$-cell function by mutated alleles of the gene encoding forkhead transcription factor Fox01. Nat. Genet. 32, 245-253

31 MacDermot, K.D., Bonora, E., Sykes, N., Coupe, A.M., Lai, C.S., Vernes, S.C., Vargha-Khadem, F., McKenzie, F., Smith, R.L., Monaco, A.P. and Fisher, S.E. (2005) Identification of FOXP2 truncation as a novel cause of developmental speech and language deficits. Am. J. Hum. Genet. 76, 1074-1080

32 Feuk, L., Kalervo, A., Lipsanen-Nyman, M., Skaug, J., Nakabayashi, K. Finucane, B., Hartung, D., Innes, M., Kerem, B., Nowaczyk, M.j. et al. (2006) Absence of a paternally inherited FOXP2 gene in developmental verbal dyspraxia. Am. J. Hum. Genet. 79, 965-972

33 Kosho, T., Sakazume, S., Kawame, H., Wakui, K., Wada, T., Okoshi, Y., Mikawa, M., Hasegawa, T., Matsuura, N., Niikawa, N. et al. (2008) De-novo balanced translocation between 7q31 and 10p14 in a girl with central precocious puberty, moderate mental retardation, and severe speech impairment. Clin. Dysmorphol. 17, 31-34

34 Shriberg, L.D., Ballard, K.J., Tomblin, J.B., Duffy, J.R., Odell, K.H. and Williams, C.A. (2006) Speech, prosody, and voice characteristics of a mother and daughter with a 7;13 translocation affecting FOXP2. J. Speech Lang. Hear. Res. 49, 500-525

35 Lennon, P.A., Cooper, M.L., Peiffer, D.A., Gunderson, K.L., Patel, A., Peters, S., Cheung, S.W. and Bacino, C.A. (2007) Deletion of 7q31.1 supports involvement of FOXP2 in language impairment: clinical report and review. Am. J. Med. Genet. A 143A, 791-798

36 Zeesman, S., Nowaczyk, M.J., Teshima, I., Roberts, W., Cardy, J.0., Brian, J., Senman, L., Feuk, L., Osborne, L.R. and Scherer, S.W. (2006) Speech and language impairment and oromotor dyspraxia due to deletion of $7 q 31$ that involves FOXP2. Am. J. Med. Genet. A 140, 509-514

37 Stroud, J.C., Wu, Y., Bates, D.L., Han, A., Nowick, K., Paabo, S., Tong, H. and Chen, L. (2006) Structure of the forkhead domain of FOXP2 bound to DNA. Structure 14, 159-166

38 Fisher, S.E. and Scharff, C. (2009) FOXP2 as a molecular window into speech and language. Trends Genet. 25, 166-177

39 Lai, C.S., Gerrelli, D., Monaco, A.P., Fisher, S.E. and Copp, A.J. (2003) FOXP2 expression during brain development coincides with adult sites of pathology in a severe speech and language disorder. Brain $\mathbf{1 2 6}$ 2455-2462

40 Groszer, M., Keays, D.A., Deacon, R.M., de Bono, J.P., Prasad-Mulcare, S., Gaub, S., Baum, M.G., French, C.A., Nicod, J., Coventry, J.A. et al. (2008) Impaired synaptic plasticity and motor learning in mice with a point mutation implicated in human speech deficits. Curr. Biol. 18, 354-362 
41 French, C.A., Groszer, M., Preece, C., Coupe, A.M., Rajewsky, K. and Fisher, S.E. (2007) Generation of mice with a conditional Foxp2 null allele. Genesis $\mathbf{4 5}, 440-446$

42 Vernes, S.C., Newbury, D.F., Abrahams, B.S., Winchester, L., Nicod, J., Groszer, M., Alarcon, M., Oliver, P.L., Davies, K.E., Geschwind, D.H. et al. (2008) A functional genetic link between distinct developmental language disorders. N. Engl. J. Med. 359, 2337-2345

43 Inda, M.C., DeFelipe, J. and Munoz, A. (2006) Voltage-gated ion channels in the axon initial segment of human cortical pyramidal cells and their relationship with chandelier cells. Proc. Natl. Acad. Sci. U.S.A. 103, 2920-2925

44 Alarcon, M., Abrahams, B.S., Stone, J.L., Duvall, J.A., Perederiy, J.V., Bomar, J.M., Sebat, J., Wigler, M., Martin, C.L., Ledbetter, D.H. et al. (2008) Linkage, association, and gene-expression analyses identify CNTNAP2 as an autism-susceptibility gene. Am. J. Hum. Genet. 82, 150-159

45 Strauss, K.A., Puffenberger, E.G., Huentelman, M.J., Gottlieb, S., Dobrin, S.E., Parod, J.M., Stephan, D.A. and Morton, D.H. (2006) Recessive symptomatic focal epilepsy and mutant contactin-associated protein-like 2. N. Engl. J. Med. 354, 1370-1377
46 The SLI Consortium (2002) A genomewide scan identifies two novel loci involved in specific language impairment. Am. J. Hum. Genet. 70, 384-398

47 Vernes, S.C., Spiteri, E., Nicod, J., Groszer, M., Taylor, J.M., Davies, K.E., Geschwind, D.H. and Fisher, S.E. (2007) High-throughput analysis of promoter occupancy reveals direct neural targets of FOXP2, a gene mutated in speech and language disorders. Am. J. Hum. Genet. 81, 1232-1250

48 Spiteri, E., Konopka, G., Coppola, G., Bomar, J., Oldham, M., Ou, J., Vernes, S.C., Fisher, S.E., Ren, B. and Geschwind, D.H. (2007) Identification of the transcriptional targets of FOXP2, a gene linked to speech and language, in developing human brain. Am. J. Hum. Genet. 81, 1144-1157

49 Vernes, S.C., MacDermot, K.D., Monaco, A.P. and Fisher, S.E. (2009) Assessing the impact of FOXP1 mutations on developmental verbal dyspraxia. Eur. J. Hum. Genet., 17, 1354-1358

50 Fisher, S.E. and Marcus, G.F. (2006) The eloquent ape: genes, brains and the evolution of language. Nat. Rev. Genet. 7, 9-20

Received 25 August 2009

doi:10.1042/BST0371263 